\title{
Magnetic Separation of Autophagosomes from Mammalian Cells Using Magnetic-Plasmonic Hybrid Nanobeads
}

\author{
Mari Takahashi, ${ }^{\dagger}$ Priyank Mohan, ${ }^{\dagger}$ Kojiro Mukai, ${ }^{\ddagger}$ Yuichi Takeda, ${ }^{\S}$ Takeo Matsumoto, \\ Kazuaki Matsumura, ${ }^{\circ}$ Masahiro Takakura, ${ }^{\perp}$ Hiroyuki Arai, ${ }^{\ddagger}$ Tomohiko Taguchi, ${ }^{\#}$ \\ and Shinya Maenosono* ${ }^{\dagger}$ (i)
}

${ }^{\dagger}$ School of Materials Science, Japan Advanced Institute of Science and Technology, 1-1 Asahidai, Nomi, Ishikawa 923-1292, Japan

${ }^{\ddagger}$ Department of Health Chemistry, Graduate School of Pharmaceutical Sciences, The University of Tokyo, 7-3-1 Hongo, Bunkyo-ku, Tokyo 113-0033, Japan

\#Pathological Cell Biology Laboratory, Graduate School of Pharmaceutical Sciences, The University of Tokyo, 7-3-1 Hongo, Bunkyo-ku, Tokyo 113-0033, Japan

${ }^{\S}$ Department of Biochemistry, Osaka University Graduate School of Medicine, 2-2 Yamadaoka, Suita, Osaka 565-0871, Japan

"Department of Obstetrics and Gynecology, Kanazawa University Graduate School of Medical Sciences, 13-1 Takaramachi, Kanazawa, Ishikawa 920-8641, Japan

${ }^{\perp}$ Department of Obstetrics and Gynecology, Kanazawa Medical University, 1-1 Daigaku, Uchinada-machi, Kahoku-gun, Ishikawa 920-0293, Japan

\section{Supporting Information}

ABSTRACT: Developments in subcellular fractionation strategies have provided the means to analyze the protein and lipid composition of organelles by proteomics. Here, we developed ultrasmall magnetic-plasmonic hybrid nanobeads and applied them to the isolation of autophagosomes by applying a magnetic field. The beads were chemically synthesized and comprised an $\mathrm{Ag} / \mathrm{FeCo} / \mathrm{Ag}$ core/shell/shell structure with a mean diameter of $15 \mathrm{~nm}$. The Ag core and the FeCo shell conferred imaging and magnetic separation capabilities, respectively. The nanobeads were transfected into mammalian cells by lipofection. Thirty minutes after lipofection, the nanobeads colocalized with Vps26 and subsequently with LC3. Cell lysates were prepared at the appropriate time points and

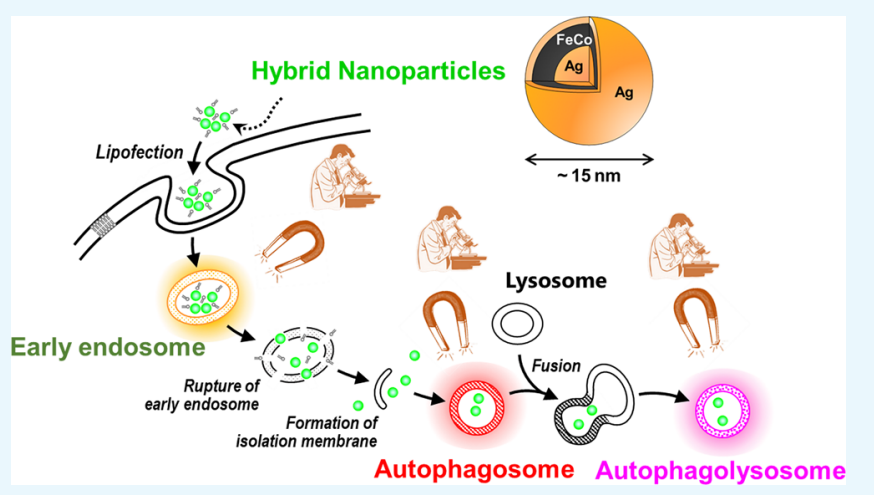
were subjected to magnetic separation. The separated fraction contained LC3-II, transferrin receptor, and LAMP2, but not LC3I, suggesting that autophagosomes engulfing endosomal origin had been isolated. The magnetic separation process was completed in less than $30 \mathrm{~min}$, providing a rapid method for isolation of autophagosomes. The present organelle isolation technique using the hybrid nanobeads with imaging and magnetic separation capabilities is highly promising for isolation of other types of organelles such as endosomes and endosome-related organelles.

\section{INTRODUCTION}

Immunomagnetic isolation is widely used to isolate cells ${ }^{1,2}$ and subcellular organelles ${ }^{3-9}$ and involves the use of magnetic beads that are coupled with cell surface or organelle-specific antibodies. In contrast to classical purification methods relying on ultracentrifugation, immunomagnetic isolation is rapid and gentle, which helps to ensure that the isolated cells and organelles remain intact. In addition to immunomagnetic isolation, magnetic beads have been used to isolate endocytic organelles, such as early endosomes ${ }^{10}$ and late endosomes, ${ }^{11}$ whereby magnetic beads are delivered into the lumen of organelles by endocytosis.

Endocytosis from the plasma membrane is mediated by various types of membrane-bound vesicles or tubules. Clathrin- coated vesicles (CCVs) were the first to be discovered ${ }^{12}$ and remain the most extensively characterized transport vesicles. ${ }^{13}$ The diameter of CCVs is $100-150 \mathrm{~nm}$, limiting the size of materials that can be packaged into this type of vesicle. Using gold particles coated with transferrin as the cargo, the effect of bead size on the efficiency of endocytosis by CCVs was previously examined. ${ }^{14}$ Indeed, this study showed that the exocytosis rate of $14 \mathrm{~nm}$ gold particles was much faster than that of $74 \mathrm{~nm}$ gold particles.

Received: July 5, 2017

Accepted: August 11, 2017

Published: August 24, 2017 
The size of commercially available magnetic beads is typically larger than $200 \mathrm{~nm}$. For example, Dynabeads (Thermo Fisher Scientific, Waltham, USA) are typically 1 to $5 \mu \mathrm{m}$ in diameter; RayBio magnetic beads (RayBiotech, Norcross, USA) are 500 $\mathrm{nm}$ in diameter; and the smallest beads, MACS MicroBeads (Miltenyi Biotec, Bergisch Gladbach, Germany), are about 50 $\mathrm{nm}$ in diameter, but when coated with antifluorescein isothiocyanate (FITC) antibodies, the hydrodynamic diameter increases to $125 \mathrm{~nm}^{15}$ Therefore, these magnetic beads are, in principle, refractory to endocytosis by CCVs.

Compared with conventional magnetic beads, we previously fabricated ultrasmall magnetic beads ( $15 \mathrm{~nm}$ in diameter) with an $\mathrm{Ag} / \mathrm{FeCo} / \mathrm{Ag}$ core/shell/shell structure. ${ }^{16}$ Although the magnetic probe had a magnetically inert $\mathrm{Ag}$ core with a mean size of $10 \mathrm{~nm}$, the magnetic beads showed separation capability. ${ }^{16}$ If the magnetic bead with the same volume of the FeCo shell was composed of iron oxide, it would be ineffective as a magnetic bead because the saturation magnetization of iron oxide $\left(400-450 \mathrm{emu} / \mathrm{cm}^{3}\right)$ is approximately a quarter that of FeCo $\left(1790 \mathrm{emu} / \mathrm{cm}^{3}\right){ }^{17}$ Therefore, we expect that our magnetic bead would be easily endocytosed and could be used as a separation probe for endosome-related organelles.

The presence of an Ag core provides visualization of the magnetic bead using a laboratory microscope. ${ }^{16}$ Metal nanoparticles, such as $\mathrm{Ag}$ and $\mathrm{Au}$ nanoparticles, have been used for cell imaging because of their intrinsic optical properties and localized surface plasmon resonance (LSPR). ${ }^{18,19}$ Furthermore, coupling of the magnetic beads and other biomolecules using strong metal-thiol interactions can be easily achieved because of the presence of an outer Ag shell compared with iron oxide particles. This type of magnetic beads will be referred to as magnetic-plasmonic hybrid nanobeads (MPNBs) hereafter.

In this study, we chose autophagosomes as target organelles which are formed in the cytoplasm in the course of autophagy among many subcellular organelles. It was reported that micrometer-sized polystyrene beads that were lipofected into mammalian cells were delivered into early endosomes where they caused damage. Then, xenophagy (a type of selective autophagy) occurred, engulfing the damaged endosomes by autophagosomes. ${ }^{20,21}$ Although the autophagosomes can engulf even micrometer-sized beads, ${ }^{21}$ we exploited this observation and applied MPNBs to isolated autophagosomes to demonstrate the capability of MPNBs for magnetic isolation of subcellular organelles.

\section{RESULTS}

Structural Characterization of MPNBs. $\mathrm{Ag} / \mathrm{FeCo} / \mathrm{Ag}$ core/shell/shell MPNBs were chemically synthesized by our previously reported method. ${ }^{16,22-24}$ Figure 1 shows transmission electron microscopy (TEM; H-7650, Hitachi, Tokyo, Japan) and scanning TEM, equipped with a high-angle annular dark-field detector (STEM-HAADF; JEM-ARM200F, JEOL, Tokyo, Japan), images of the as-synthesized MPNBs. The MPNBs were spherical in shape and a distinct core-shell structure could be observed. The average diameter of MPNBs was calculated to be about $15 \mathrm{~nm}$, with the standard deviation of the size distribution typically being around $16 \%{ }^{16,23,24}$ Figure $1 \mathrm{D}-\mathrm{G}$ shows the energy-dispersive X-ray spectroscopy (EDS) elemental mapping images for $\mathrm{Ag}, \mathrm{Fe}$, and $\mathrm{Co}$ and a merged image of these elements. It was evident from these images that the MPNBs have a distinct Ag core and FeCo shell. Figure $1 \mathrm{H}$ shows the EDS line profile, depicted by a yellow line in Figure 1G. The Ag signal clearly increased at the edge of the
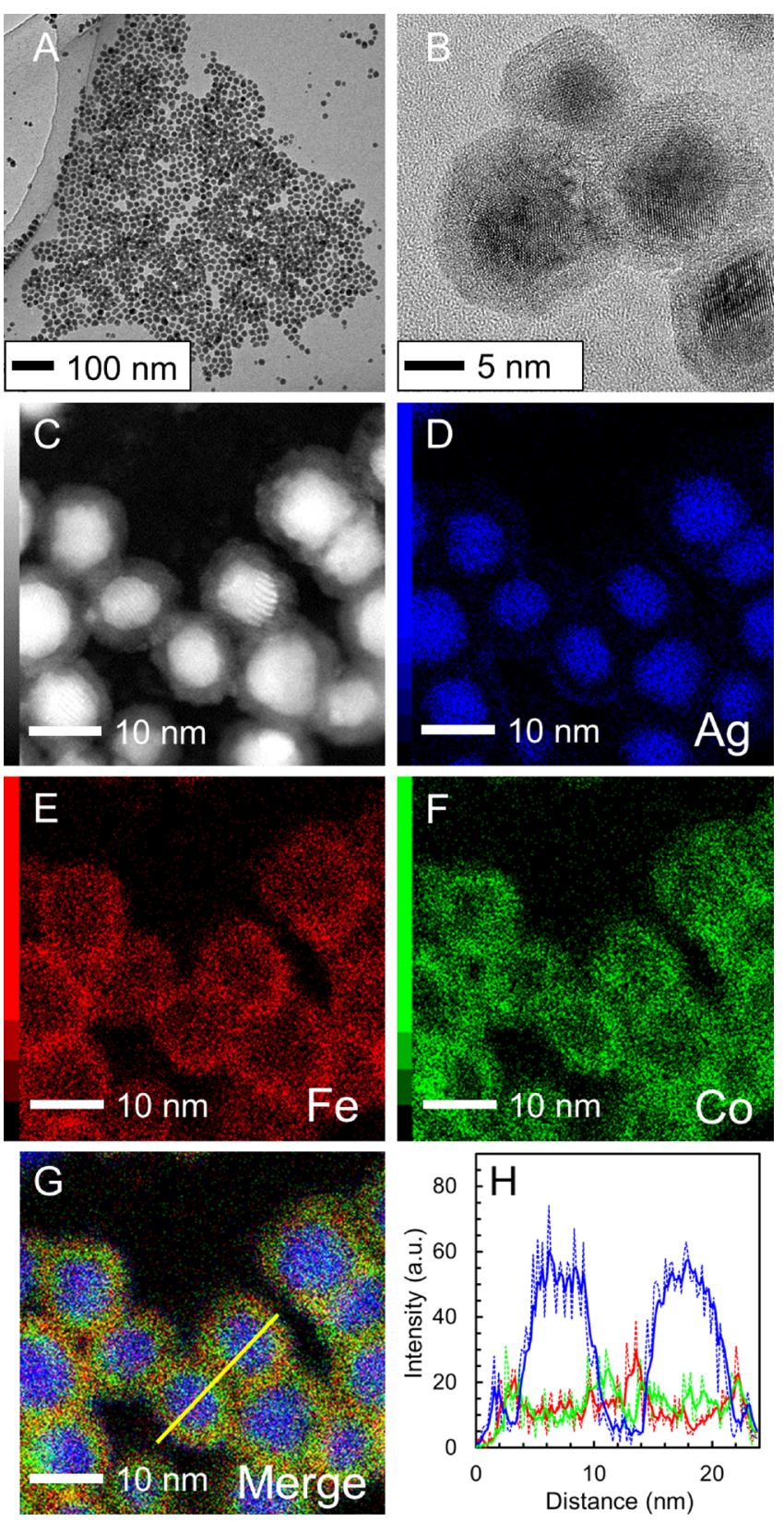

Figure 1. Morphology of the MPNBs. (A) TEM, (B) HR-TEM, and (C) STEM-HAADF images of $\mathrm{Ag} / \mathrm{FeCo} / \mathrm{Ag}$ MPNBs. (D) Ag L-edge, (E) Fe K-edge, (F) Co K-edge, and (G) merged images of EDS elemental mapping images for $\mathrm{Ag} / \mathrm{FeCo} / \mathrm{Ag}$ MPNBs. (H) EDS line profile along with the yellow line in $(\mathrm{G})$. The dashed and solid lines are the raw and low-pass-filtered profiles, respectively. The blue, green, and red colors represent $\mathrm{Ag} \mathrm{L}$-, Co K-, and Fe K-edge intensities, respectively.

MPNBs, confirming the presence of a thin Ag outer shell. The MPNBs exhibited superparamagnetic behavior at $300 \mathrm{~K}$ with a saturation magnetization value of typically around $33 \mathrm{emu} /$ g. ${ }^{16,23,24}$

Surface Modification of MPNBs. Because the surfaces of the as-synthesized MPNBs are covered with hydrophobic organic ligands, we needed to modify the surfaces to make them water-dispersible and biocompatible. $\varepsilon$-Poly-L-lysine (PLL) is a polymer of L-lysine in which the $\varepsilon$-amino and $\alpha$ carboxyl groups are condensed to make an amide bond. Because PLL is water-soluble and relatively nontoxic compared 

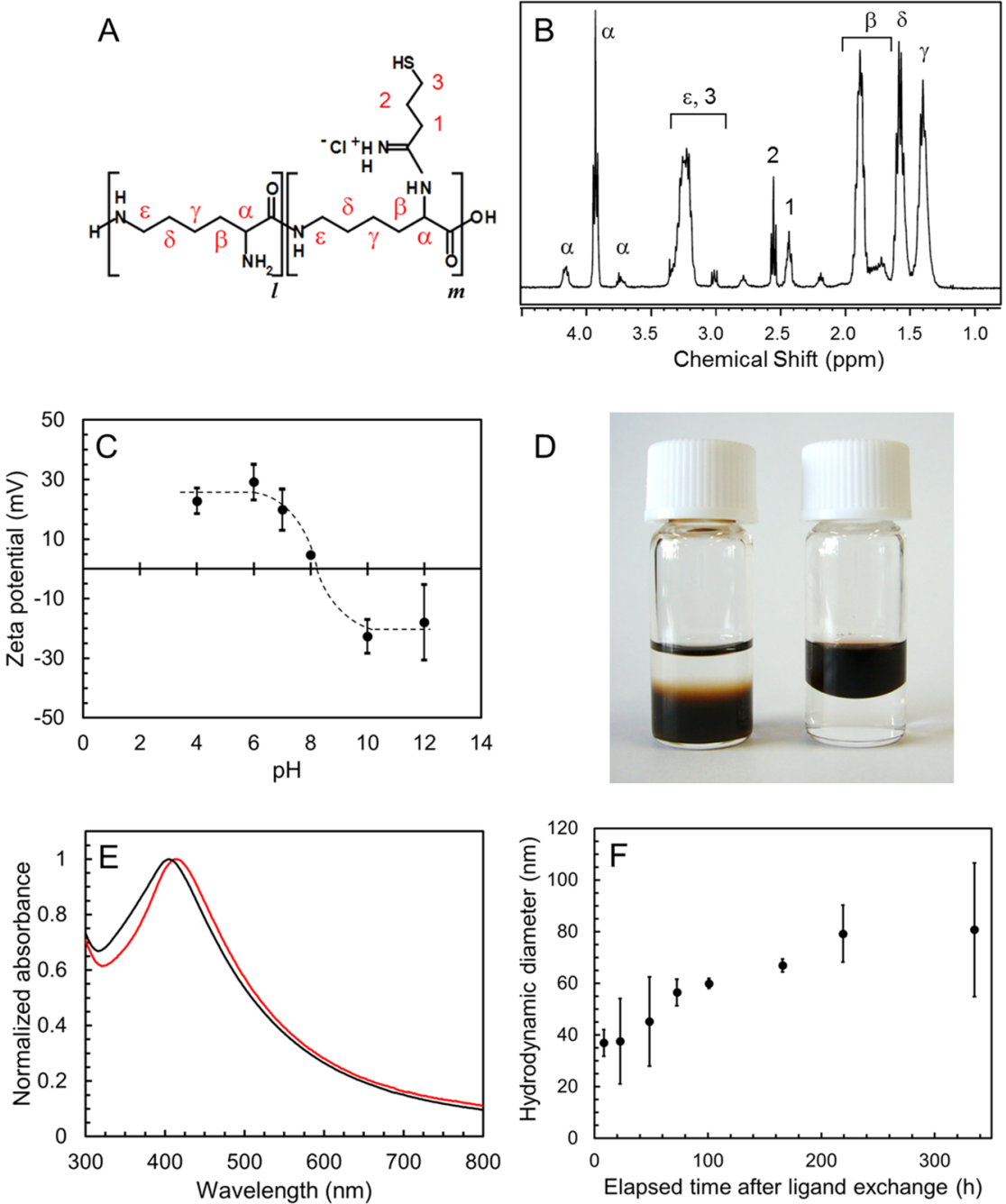

Figure 2. Structure and properties of the PLL-SH polymer. (A) Structure, (B) ${ }^{1} \mathrm{H}$ NMR spectrum, and (C) zeta potential of the PLL-SH polymer. The ratio of $l / m$ is 87:13. (D) Photograph of $\mathrm{Ag} / \mathrm{FeCo} / \mathrm{Ag}$ MPNBs before (right) and after (left) ligand exchange. The upper phase is hexane, and the bottom phase is water. (E) UV-vis spectra of MPNBs before (black) and after (red) ligand exchange. (F) Hydrodynamic diameter of MPNBs plotted as a function of elapsed time after ligand exchange.

with $\alpha$-poly-L-lysine, its derivatives have been applied in food preservatives, emulsifying agents, dietary agents, biodegradable fibers, highly water absorbable hydrogels, drug carriers, and cryopreservation agents. ${ }^{25,26}$ In this study, 2-iminothiolane was made to react with the amino group in the side chain of PLL to obtain partially (theoretical substitution rate of $20 \%$ ) thiolated PLL (PLL-SH). Figure 2A,B shows the structure and proton nuclear magnetic resonance $\left({ }^{1} \mathrm{H}\right.$ NMR) spectrum of PLL-SH (actual substitution rate was determined to be $13 \%$ ).

Figure $2 \mathrm{C}$ shows the $\mathrm{pH}$ dependence of the zeta potential of PLL-SH dissolved in water. At $\mathrm{pH} 7$, the zeta potential of PLL$\mathrm{SH}$ was around $+20 \mathrm{mV}$. There are several possible reasons for the negative zeta potential under alkaline conditions. One possible reason is the proton dissociation of thiol groups; another is that the protonated amino groups are surrounded by excess counter ions. ${ }^{27}$ The surface of MPNBs were modified with PLL-SH via a ligand exchange reaction; then, MPNBs could be readily dispersed in water as shown in Figure 2D. The zeta potential of PLL-SH-modified MPNBs dispersed in water was measured to be about $+42 \mathrm{mV}$ (the $\mathrm{pH}$ value was found to be 6). When the $\mathrm{pH}$ value was adjusted to be 7 by adding
$\mathrm{NaOH}$ solution, the zeta potential of PLL-SH-modified MPNBs was measured to be $+7 \mathrm{mV}$.

Figure 2E shows ultraviolet-visible (UV-vis) spectra of MPNBs before and after ligand exchange. The UV-vis spectrum of MPNBs before ligand exchange showed the LSPR peak at $405 \mathrm{~nm}$. After ligand exchange, the LSPR peak of water-dispersible MPNBs red-shifted from 405 to $415 \mathrm{~nm}$. Typically, the LSPR peak wavelength linearly increases with increasing the refractive index of the surrounding medium. Therefore, the red shift of the LSPR peak is presumably due to the ligand exchange with PLL-SH which has the higher refractive index than hexane, water, and hydrophobic organic ligands. Because the extinction of the LSPR band is linearly proportional to the concentration of $\mathrm{MPNBs},{ }^{16}$ one can precisely estimate the concentration of MPNBs from the UV-vis spectrum. The concentration of the undiluted water dispersion of MPNBs was estimated to be $5.2 \mathrm{mg} / \mathrm{mL}$ from the UV-vis spectrum and was used in the following cellular experiment.

Colloidal stability of PLL-SH-modified MPNBs dispersed in water was investigated by measuring the hydrodynamic size of MPNBs as a function of elapsed time after ligand exchange as 
shown in Figure 2F. The hydrodynamic diameter of MPNBs slightly increased from $37 \pm 5$ to $81 \pm 26 \mathrm{~nm}$ within 14 days.

Lipofection of MPNBs into COS-1 Cells. PLL-SHmodified MPNBs were transfected into COS-1 cells by lipofection. At an appropriate time after lipofection, the cells were fixed, stained for Vps26 (an early endosomal marker protein) or LC3 (an autophagosomal marker protein), and examined by a confocal laser scanning microscope (CLSM; FV 1000D, Olympus, Tokyo, Japan). Thirty-minutes after transfection, the MPNBs partly colocalized with Vps26 (Figure 3A),
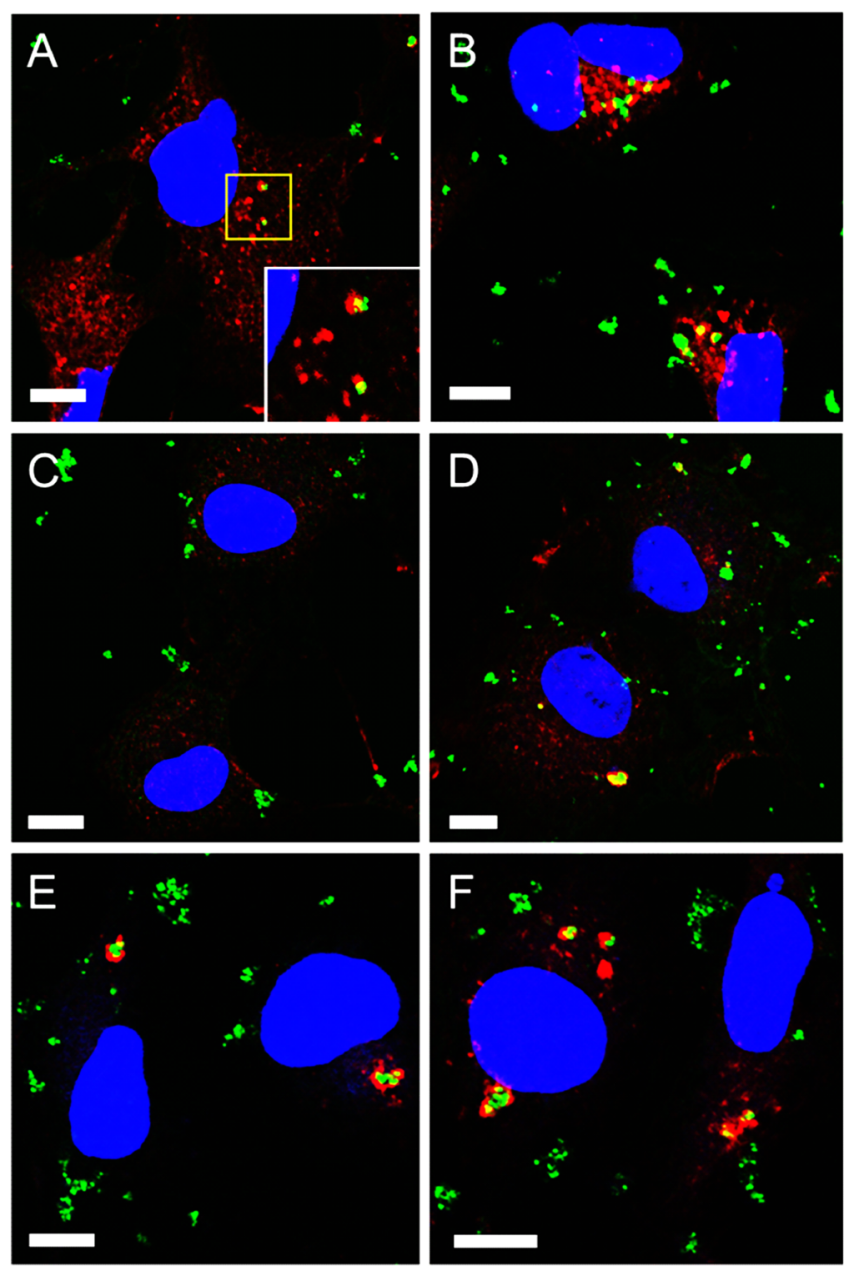

Figure 3. CLSM images of $\mathrm{Ag} / \mathrm{FeCo} / \mathrm{Ag}$ MPNB-transfected COS-1 cells. After incubation for (A) $30 \mathrm{~min}$ and (B) $1 \mathrm{~h}$ for Vps26 staining, and (C) $30 \mathrm{~min},(\mathrm{D}) 1 \mathrm{~h},(\mathrm{E}) 2 \mathrm{~h}$, and (F) $4 \mathrm{~h}$ for LC3 staining. Blue and green colors represent 4',6-diamidino-2-phenylindole (DAPI)stained nuclei and $\mathrm{Ag} / \mathrm{FeCo} / \mathrm{Ag} \mathrm{MPNBs}$, respectively. Red color represents Vps26 $(\mathrm{A}, \mathrm{B})$ or LC3 $(\mathrm{C}-\mathrm{F})$. All scale bars correspond to $10 \mu \mathrm{m}$. The inset shown at the bottom right of panel (A) is a magnified view of the region enclosed by the yellow line in panel (A).

but not with LC3 (Figure 3C). One hour after lipofection, there emerged a few MPNB-positive structures that were surrounded by LC3 (Figure 3D). Those structures were mostly evident 2 and $4 \mathrm{~h}$ after lipofection (Figure 3E,F). These results indicated that the MPNBs were first delivered into early endosomes and then targeted to autophagosomes. Note that the imaging of MPNBs was achieved by utilizing plasmon scattering (not fluorescence) in the present study. Because it has been known that $\mathrm{Ag}$ nanoparticles can become fluorescent only when their diameter is smaller than typically $2.5 \mathrm{~nm},{ }^{28-30}$ the $10 \mathrm{~nm} \mathrm{Ag}$ core is not fluorescent, but can act as an efficient scatterer because the scattering cross section of $\mathrm{Ag}$ nanoparticles is more than 1 order of magnitude higher than that of Au nanoparticles. ${ }^{31}$

Next, we examined the COS-1 cells lipofected with MPNBs by TEM (JEM-1200 EX, JEOL, Tokyo, Japan). The TEM images of the cells for $30 \mathrm{~min}$ and $2 \mathrm{~h}$ after the lipofection of MPNBs are shown in Figure 4; at these same time points, the MPNBs were shown to be surrounded by Vps26 (Figure 3A) or LC3 (Figure 3E) by immunostaining. We detected the electron-dense granular substances within membrane-bound structures. Since the mean diameter of MPNBs is about $15 \mathrm{~nm}$, the electron-dense granular substances were considered to be a complex assembly between the transfection reagent (Lipofectamine 2000) and MPNBs. In fact, as shown in the inset of Figure 4D, individual MPNBs can be clearly seen in the highcontrast magnified view of the electron-dense granular substance. As shown in Figure 4A-C, some MPNBs were found to be incorporated in single (endosome-like) or double (autophagosome-like) membrane compartments in the case of the cells for $30 \mathrm{~min}$ after the lipofection of MPNBs. As shown in Figure 4D-F, on the other hand, most of MPNBs seemed to be incorporated in autophagolysosome-like structures in the case of the cells for $2 \mathrm{~h}$ after the lipofection of MPNBs.

Magnetic Separation of Autophagosomes. An overview of the magnetic separation protocol is depicted in Figure 5A. The cells lipofected with MPNBs were detached from the culture dish using a cell lifter at the appropriate time and homogenized using a Dounce tissue grinder (Wheaton Industries Inc., Millville, USA). The resultant cell lysates were subjected to magnetic separation using an autoMACS Pro Separator (Miltenyi Biotec, Bergisch Gladbach, Germany). The magnetically separated fraction was then eluted from the column, briefly spun, and resuspended in phosphate-buffered saline (PBS). The cell lysate and magnetically separated (MS) fractions were subjected to sodium dodecyl sulfate polyacrylamide gel electrophoresis (SDS-PAGE) and blotted for LC3, transferrin receptor ( $\mathrm{TfnR}$, an endosomal protein), lysosome-associated membrane protein 2 (LAMP2), and cytosolic protein [glyceraldehyde 3-phosphate dehydrogenase $(\mathrm{GAPDH})]$. As shown in Figure 5B, at time 0, the proteins of interest were not detected in the MS fraction. After $15 \mathrm{~min}$, LC3-II (a membrane-bound form of LC3), TfnR, and LAMP2, but not GAPDH, were detected in the MS fraction. The level of LC3-II and LAMP2 in the MS fractions increased up to $8 \mathrm{~h}$, whereas that of TfnR plateaued after $2 \mathrm{~h}$. Throughout the separation experiments, GAPDH and LC3-I (a cytosolic form of LC3) were not detected in the MS fractions. These results indicated that at early time points $(0.5<t<2 \mathrm{~h}$, where $t$ denotes the incubation time), autophagosomes were mainly isolated and, by contrast, at a later time point $(t>2 \mathrm{~h})$, autophagolysosomes were isolated.

\section{DISCUSSION}

In the present study, we fabricated nanobeads that have magnetic and imaging capabilities. We then applied these beads to isolate autophagosomes, exploiting a previous observation that lipofected latex bead-induced xenophagy of damaged early endosomes. ${ }^{20,21}$ The MS fraction contained LC3-II, TfnR, and LAMP2 but not LC3-I, indicating that autophagosomes/ autophagolysosomes were successfully isolated. The isolation process essentially required two steps (homogenization and magnetic separation) and took less than $30 \mathrm{~min}$ to complete. 

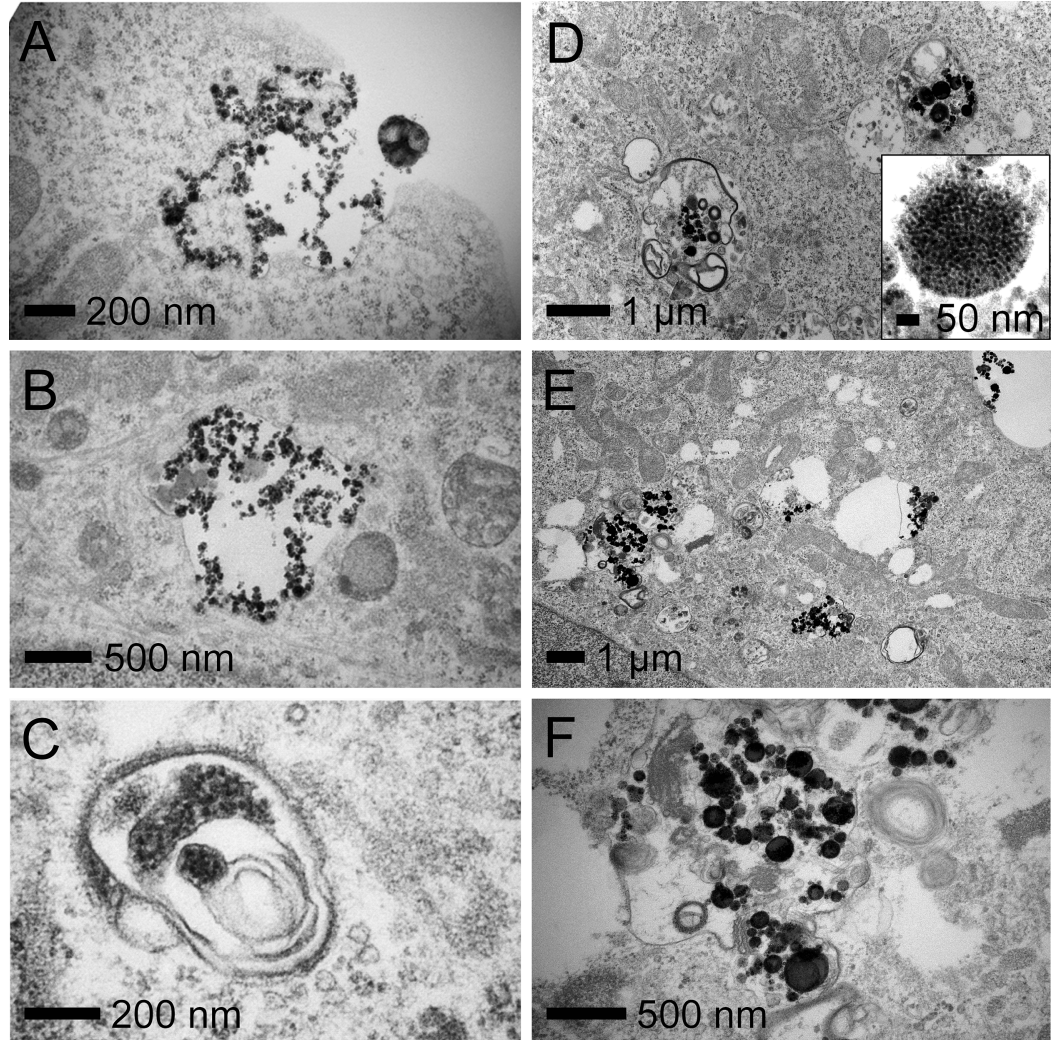

Figure 4. TEM images of $\mathrm{Ag} / \mathrm{FeCo} / \mathrm{Ag}$ MPNB-transfected COS-1 cells after $30 \mathrm{~min}(\mathrm{~A}-\mathrm{C})$ and $2 \mathrm{~h}(\mathrm{D}-\mathrm{F})$ incubation. The inset of (D) is a magnified view of a complex assembly between transfection reagent and MPNBs. Each small black dot is a single $\mathrm{Ag} / \mathrm{FeCo} / \mathrm{Ag} \mathrm{MPNB}$.

A
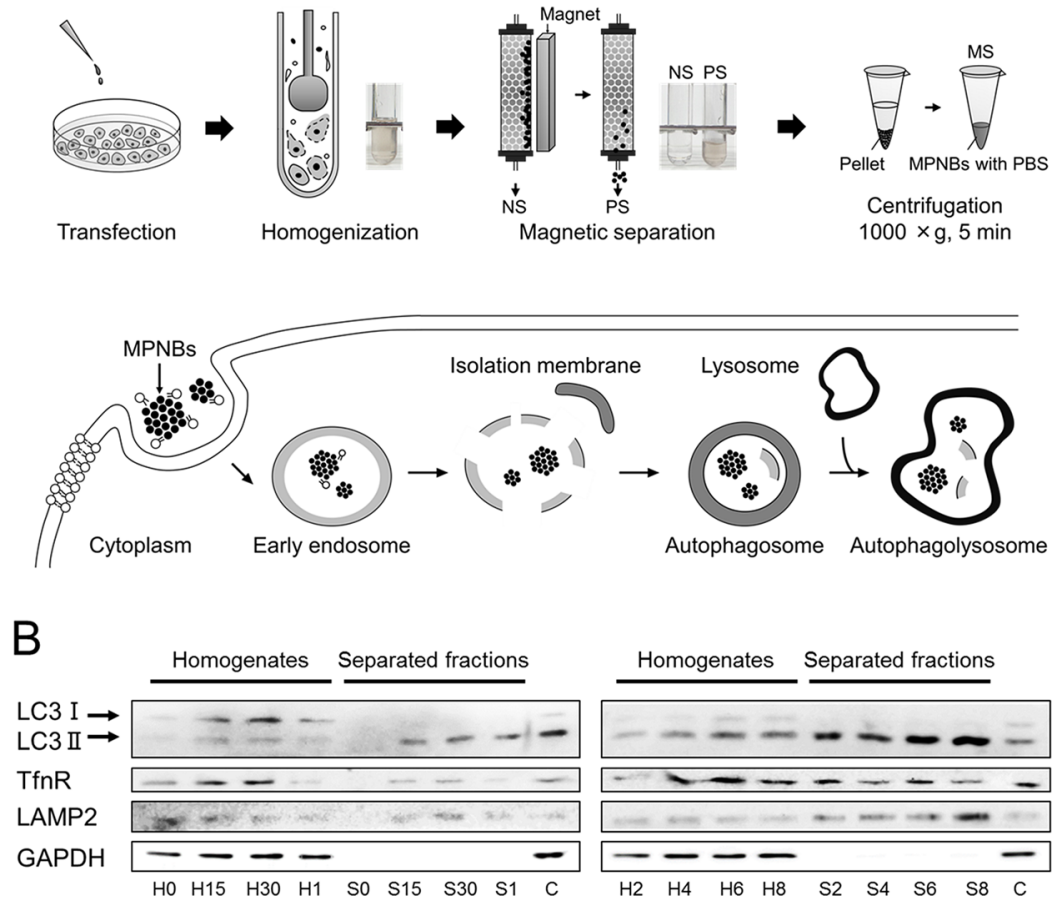

Figure 5. Magnetic separation scheme and the results of western blotting. (A) Schematic illustrations of (top) the magnetic separation protocol and (bottom) the change in the localization of MPNBs in a cell. (B) Incubation time dependence of the presence of marker proteins in the homogenate (designated as $\mathrm{H}$ ) and the separated fractions (MS, designated as S). C is a control in which the transfection reagent was present but MPNBs were absent. The number added after the initial of the sample ( $\mathrm{H}$ or $\mathrm{S}$ ) denotes the incubation time, that is, 0,15 , and 30 correspond to 0,15 , and 30 min incubations, respectively, whereas $1,2,4,6$, and 8 correspond to $1,2,4,6$, and 8 h incubations, respectively. 
Rapidly isolated autophagosomes/autophagolysosomes may lose less peripherally associated proteins, and proteomic/ lipidomic analysis should therefore provide valuable information on the molecules that are involved in autophagosome generation and maturation into autophagolysosomes. There are several biochemical methods to prevent fusion between autophagosomes and lysosomes to improve the purity of autophagosomes in ultracentrifugation ${ }^{32,33}$ or immunomagnetic isolation. ${ }^{34}$ By combining these biochemical methods with the present magnetic separation technique using the MPNBs, it will enable a swift and mild purification of autophagosomes formed in xenophagy.

The differences between the present MPNBs and conventional magnetic probes are summarized as follows regarding magnetic property, size, imaging capability, and functionalization. The saturation magnetization of $\mathrm{MPNBs}^{24}$ normalized to a single particle volume is about $250 \mathrm{emu} / \mathrm{cm}^{3}$, which is almost comparable to that of iron oxide nanoparticles of the same size. Although an MPNB contains a $10 \mathrm{~nm} \mathrm{Ag}$ core corresponding to $30 \%$ of the volume of the bead, the reason why MPNBs show comparable magnetic properties to iron oxide is that the saturation magnetization of FeCo is 4 times higher than that of iron oxide. As shown in the photograph in Figure 5, most MPNBs were efficiently separated, and thus the positive selection (PS) was brown, whereas the negative selection (NS) was colorless.

The smallest-sized conventional magnetic bead is about 50 $\mathrm{nm}$ in diameter (MACS MicroBeads, Miltenyi Biotec). The hydrodynamic diameter of FITC antibody-conjugated MACS MicroBeads dispersed in PBS is $125 \mathrm{~nm}^{15}$ whereas that of MPNBs dispersed in water is much smaller at $37 \mathrm{~nm}(81 \mathrm{~nm}$ even after 14 days from ligand exchange). When the MPNBs were mixed with the transfection reagent in Opti-MEM (which corresponds to the conditions just before lipofection of MPNBs into cells), the hydrodynamic diameter of MPNBs was found to increase up to $1352 \pm 182 \mathrm{~nm}$ as shown in Figure S1 (Supporting Information). This result clearly indicates that complex assemblies between the transfection reagent and MPNBs are formed, which are consistent with the TEM observations (Figure 4).

Cell cytotoxicity is also an important issue when working with cells. Therefore, we performed additional experiments to investigate cell cytotoxicity of the MPNBs as shown in Figure S2 (Supporting Information). The COS-1 cells were treated with 5,10 , and $20 \mu \mathrm{g} / \mathrm{mL}$ of MPNBs for 1,2 , and $4 \mathrm{~h}$, respectively. The viability was measured with the intracellular esterase activity. The results showed no obvious dosedependent decrease of cell viability, whereas the timedependent decrease of cell viability was clearly observed. However, when the treatment time was shorter than $2 \mathrm{~h}$, the cell viability was still higher than $70 \%$, and thus there seems to be no significant influence on the isolation of autophagosomes because autophagosomes were isolated at early time points $(0.5$ $<t<2 \mathrm{~h}$ ). Note that the concentration of MPNBs used in the transfection experiments was $6.7 \mu \mathrm{g} / \mathrm{mL}$.

The striking feature of the MPNBs is the imaging capability. To examine the localization of the beads in a cell, TEM observations or modification of the beads with fluorescent dyes was generally required. The MPNBs require neither TEM observations nor modification with fluorescent dyes because of their intrinsic optical properties (plasmon scattering). Because plasmon scattering is observed semipermanently, it can be used robustly even under severe conditions regardless of environ- mental changes. Plasmonic properties of MPNBs can be utilized not only for imaging but also for sensing. For example, the $\mathrm{pH}$ of the environment where the nanoparticles are localized could be measured using surface-enhanced Raman scattering (SERS) spectroscopy. ${ }^{35}$ The spatially resolved imaging of the $\mathrm{pH}$ in live cells could be achieved using gold nanoparticles as probes and 4-mercaptobenzoic acid as the Raman reporter. The SERS spectrum of the Raman reporter changed depending on the surrounding $\mathrm{pH}$. Therefore, the MPNBs presented here can also be used as SERS probes to quantitatively determine the $\mathrm{pH}$ in the luminal space of endosomes/endosome-related organelles.

Because the surface of the MPNBs used in this study is covered with PLL-SH, similar to the polymer-coated iron oxide particles, the MPNBs are readily conjugated to proteins or biomolecules through an amino group in PLL. Ligandconjugated MPNBs can be delivered to specific compartments, such as recycling endosomes ${ }^{36}$ and the trans-Golgi network, ${ }^{37}$ which allows the isolation of these compartments for in vitro functional assays and proteomics studies in the future.

\section{CONCLUSIONS}

In this study, monodispersed $\mathrm{Ag} / \mathrm{FeCo} / \mathrm{Ag}$ core/shell/shell MPNBs with a mean diameter of $15 \mathrm{~nm}$ were chemically synthesized, and then the nanobeads were transfected into the COS-1 cells by lipofection. After incubation for a finite period of time $(0-8 \mathrm{~h})$, the localization of nanobeads in a cell was visualized by confocal microscopy using plasmon scattering of Ag cores. The nanobeads reached early endosomes within 30 min, and subsequently, the nanobeads became positive with LC3. Then, the autophagosomes containing the nanobeads were successfully isolated by the magnetic separation technique. This process took only $\sim 30 \mathrm{~min}$ after cell lysis, providing a swift way to isolate autophagosomes. In consequence, the capability of MPNBs for magnetic isolation of subcellular organelles is clearly demonstrated, and it is highly promising for isolation of other types of organelles such as endosomes/ endosome-related organelles, the trans-Golgi network, and even CCVs.

\section{EXPERIMENTAL SECTION}

Chemicals Used in the Fabrication of the WaterDispersible MPNBs. The reagents cobalt acetylacetonate $\left[\mathrm{Co}(\mathrm{acac})_{2}\right.$, purity $\left.97 \%\right]$, iron acetylacetonate $\left[\mathrm{Fe}(\mathrm{acac})_{3}\right.$, purity $\geq 99.9 \%$, silver nitrate $\left(\mathrm{AgNO}_{3}\right.$, purity $\left.\geq 99.9999 \%\right)$, 1,2-hexadecanediol (purity 90\%), sodium hydroxide $(\mathrm{NaOH}$, purity $\geq 98 \%$ ), oleylamine (OLA, purity $70 \%$ ), oleic acid (OA, purity $90 \%$ ), and tetraethylene glycol (TEG, purity 99\%) were purchased from Sigma-Aldrich (St. Louis, USA) and were used as received. PLL was purchased from JNC Co. (Tokyo, Japan), and 2-iminothiolane and ethanol were purchased from Nacalai Tesque (Kyoto, Japan). Acetone and hexane were purchased from Kanto Chemical (Tokyo, Japan), and toluene and hydrochloric acid $(\mathrm{HCl})$ were purchased from Wako Pure Chemical (Osaka, Japan).

Chemicals for Cellular Experiments. PermaFluor aqueous mounting medium, page ruler prestained protein ladder, Lipofectamine 2000, and Opti-MEM were purchased from Thermo Fisher Scientific (Waltham, USA). Ammonium chloride, digitonin, and 2-mercaptoethanol were purchased from Wako Pure Chemical. Laemmli sample buffer $(2 \times)$ and Tris-buffered saline were purchased from Bio-Rad (Hercules, 
USA). Poly-L-lysine solution $[0.1 \%(\mathrm{w} / \mathrm{v})]$, bovine serum albumin (BSA), and Dulbecco's modified Eagle's medium (DMEM) were purchased from Sigma-Aldrich. Paraformaldehyde (4\%) PBS and polyoxyethylene sorbitan monolaurate (TWEEN20) were purchased from Nacalai Tesque. Amersham ECL select western blotting detection reagent was purchased from GE Healthcare (Little Chalfont, UK). N-Cyclohexyl-3aminopropanesulfonic acid (CAPS) was purchased from Dojindo Molecular Technologies Inc. (Kumamoto, Japan), and acrylamide/bis solution [30\% (w/v)] was purchased from Cosmo Bio Co., Ltd. (Tokyo, Japan).

For observations by CLSM, mouse antiLC3 antibody and goat antiVPS26A antibody were purchased from Cosmo Bio Co., Ltd. and Everest Biotech (Upper Heyford, UK), respectively. As secondary antibodies, Alexa Fluor 594 antimouse IgG and Alexa Fluor 594 goat-mouse IgG were purchased from Thermo Fisher Scientific. For western blot analysis, rabbit antiLC3 was purchased from Medical and Biological Laboratories (Nagoya, Japan). Mouse antiGAPDH, mouse antiLAMP2, and mouse antiTfnR were purchased from Merck Millipore (Darmstadt, Germany), Cosmo Bio Co., Ltd., and Thermo Fisher Scientific, respectively. Horseradish peroxidase-conjugated antimouse IgG antibody and antirabbit IgG antibody were purchased from GE Healthcare.

Synthesis of $\mathrm{Ag} / \mathrm{FeCo} / \mathrm{Ag}$ MPNBs. The synthetic scheme for $\mathrm{Ag} / \mathrm{FeCo} / \mathrm{Ag} \mathrm{MPNBs}$ has been described in our previous papers. ${ }^{16,22-24}$ Briefly, $0.1 \mathrm{mmol}$ of $\mathrm{AgNO}_{3}, 10 \mathrm{mmol}$ of OLA, 8 mmol of OA, and $10 \mathrm{~mL}$ of TEG were placed into a three-neck flask, which was connected to a condenser attached to a trap sphere. After Ar bubbling at room temperature for $5 \mathrm{~min}$, the temperature was increased to $100{ }^{\circ} \mathrm{C}$ and maintained at this temperature for $10 \mathrm{~min}$. Then, the temperature was further increased to $250{ }^{\circ} \mathrm{C}$. During this increase in temperature, the first stock solution containing $0.2 \mathrm{mmol}$ of $\mathrm{Fe}(\mathrm{acac})_{3}, 0.2 \mathrm{mmol}$ of $\mathrm{Co}(\mathrm{acac})_{2}, 1 \mathrm{~mL}$ of OLA, and $2 \mathrm{~mL}$ of toluene was injected while maintaining the temperature. Once $250{ }^{\circ} \mathrm{C}$ had been reached, the second stock solution containing $0.1 \mathrm{mmol}$ of $\mathrm{AgNO}_{3}, 1 \mathrm{~mL}$ of OLA, and $1 \mathrm{~mL}$ of toluene was injected into the reaction solution. Immediately after the second injection, the temperature was decreased to $230^{\circ} \mathrm{C}$ within $15 \mathrm{~min}$. Then, the reaction solution was cooled down following a washing process. The reaction solution was divided into two centrifuge tubes. Then, acetone was added to a final volume of $45 \mathrm{~mL}$, following centrifugation at $3760 \mathrm{~g}$ for $5 \mathrm{~min}$. After discarding the supernatant, $400 \mu \mathrm{L}$ of hexane was added into two tubes to disperse the MPNBs. Then, $200 \mu \mathrm{L}$ of dispersion was transferred into new tubes, and acetone was added to the four tubes to make final volumes of $45 \mathrm{~mL}$. The four tubes were centrifuged under the same conditions, and the MPNBs were obtained after discarding the supernatant. The MPNBs were kept in a vacuum drying system. The as-synthesized $\mathrm{Ag} / \mathrm{FeCo} /$ $\mathrm{Ag}$ MPNBs were characterized by TEM operated at $100 \mathrm{kV}$, EDS-attached STEM-HAADF detector operated at $200 \mathrm{kV}$ with a spherical aberration corrector and a nominal resolution of $0.8 \AA$ and UV-vis spectroscopy (V-750, Jasco, Tokyo, Japan).

Synthesis of the PLL-SH Polymer. PLL aqueous solution $(10 \mathrm{mmol}, 25 \mathrm{wt} \%)$ was placed in a glass vial, and the $\mathrm{pH}$ was adjusted to 7 by adding a small amount of $\mathrm{HCl}$; then, $2 \mathrm{mmol}$ of 2 -iminothiolane was added to give a substitutional ratio of $20 \%$ for the thiol group. The aqueous solution was mixed using a magnetic stirrer bar for $2 \mathrm{~h}$ at room temperature. Then, the products were obtained following dialysis for 2 days using the dialysis membrane (molecular weight cutoff of 500) and freezedrying for 2 days. The substitutional ratio of the thiol group was calculated from the ${ }^{1} \mathrm{H}$ NMR spectrum of the polymer sample. The ${ }^{1} \mathrm{H}$ NMR spectrum of PLL-SH dissolved in $\mathrm{D}_{2} \mathrm{O}$ was recorded at $25{ }^{\circ} \mathrm{C}$ using a $400 \mathrm{MHz} \mathrm{NMR}$ spectrometer (BioSpin AVANCE III, Bruker, Billerica, USA). The zeta potential of PLL-SH was determined by plotting the zeta potential against $\mathrm{pH}$. The zeta potential of PLL-SH dissolved in pure water $(1 \mathrm{mg} / \mathrm{mL})$ was measured using a Zetasizer Nano ZS ZEN3600 (Malvern Instruments Ltd., Malvern, UK) instrument. To tune the $\mathrm{pH}$ of the polymer solution $(1 \mathrm{~mL})$, a small amount of $\mathrm{HCl}$ or $\mathrm{NaOH}$ was added. These measurements were repeated several times, and the average and standard deviation were estimated for each $\mathrm{pH}$.

Ligand Exchange of the MPNBs. The as-synthesized MPNBs were capped with hydrophobic molecules such as OLA and $\mathrm{OA}$; hence, they could only be dispersed in a nonpolar solvent. To make them water-soluble, the capping ligands on the surface of the MPNBs were exchanged with PLL-SH. The precipitate of MPNBs in two tubes was dispersed in $200 \mu \mathrm{L}$ of hexane. Then, $10 \mathrm{mg}$ of PLL-SH was dispersed in $1 \mathrm{~mL}$ of pure water, and $100 \mu \mathrm{L}$ of ethanol was added to the PLL-SH aqueous solution. The hexane dispersion of MPNBs was added to the PLL-SH aqueous solution, followed by sonication for 30 min to enhance the phase-transfer reaction at the interface between the water and hexane phases. During sonication, more pure water $(5 \mathrm{~mL})$ was added. The mixture was transferred into six $1.5 \mathrm{~mL}$ tubes, and the total volume of each tube was made up to $1 \mathrm{~mL}$; then, the tubes were centrifuged twice at $54879 \mathrm{~g}$ for 3 min (himac CS100FNX, Hitachi, Tokyo, Japan). Finally, a small amount of pure water was added to redisperse the MPNBs. Then, all MPNBs were collected in one tube. The concentration of the $\mathrm{Ag} / \mathrm{FeCo} / \mathrm{Ag}$ MPNBs was determined by measuring the UV-vis spectrum and using the master curve of $y=0.024 x[y$ : extinction intensity, $x$ : concentration of the MPNBs $(\mu \mathrm{g} / \mathrm{mL})]$. The zeta potential and hydrodynamic diameter of PLL-SH-modified MPNBs dispersed in pure water were measured using a Zetasizer Nano ZS ZEN3600 (Malvern Instruments Ltd., Malvern, UK) instrument.

CLSM Imaging. Sterilized coverslips of $12 \mathrm{~mm}$ diameter (Matsunami, Osaka, Japan) were treated with $0.01 \%(\mathrm{w} / \mathrm{v})$ poly-L-lysine aqueous solution for $10 \mathrm{~min}$. Then, after discarding the solution the coverslips were dried and washed three times in PBS. The coverslips were then stored in PBS at room temperature before use. The COS- 1 cells $(20000)$ in 0.5 $\mathrm{mL}$ of DMEM containing 10\% fetal bovine serum (FBS) were seeded into each well of a 24 -well plate in which a poly-L-lysinecoated coverslip had been placed. Then, the cells were incubated overnight at $37{ }^{\circ} \mathrm{C}$. The next day, $1 \mu \mathrm{L}$ of Lipofectamine 2000 reagent was mixed with $50 \mu \mathrm{L}$ of OptiMEM. After $5 \mathrm{~min}, 50 \mu \mathrm{L}$ of Opti-MEM containing $4 \mu \mathrm{g}$ of MPNBs was added, and the mixture was incubated for $20 \mathrm{~min}$. Then, $100 \mu \mathrm{L}$ of the mixture was added into each well, and the cells and MPNBs were incubated for $30 \mathrm{~min}, 1,2$, and $4 \mathrm{~h}$. After incubation, the supernatant was removed and washed with PBS. The cells were fixed with 4\%-paraformaldehyde phosphate buffer solution for $15 \mathrm{~min}$ and then washed with PBS. The cells were next permeabilized with $50 \mu \mathrm{g} / \mathrm{mL}$ digitonin-PBS for 5 min and then washed with PBS. Quenching was then performed with $50 \mathrm{mM} \mathrm{NH} \mathrm{m}_{4} \mathrm{Cl}-\mathrm{PBS}$ for $10 \mathrm{~min}$, followed by a PBS wash. The sample was blocked with 3\% BSA-PBS for 30 min at room temperature. After discarding the blocking solution, primary antibody in 3\% BSA-PBS (for LC3 and 
Vps26) was added, and the samples were incubated at $4{ }^{\circ} \mathrm{C}$ overnight. The next day, the sample was washed with PBS for 5 min three times and then treated with the secondary antibody (Alexa Fluor 594 antimouse IgG and Alexa Fluor 594 antigoat $\mathrm{IgG}$ ) and $300 \mathrm{nM}$ DAPI in 3\% BSA-PBS for $1 \mathrm{~h}$ at room temperature. The sample was then washed three times with PBS for $5 \mathrm{~min}$. The glass coverslip was carefully removed and placed on the mount agent on a glass substrate for CLSM (FV 1000D, Olympus, Tokyo, Japan) observation. To observe the samples, laser wavelengths of 405 and $559 \mathrm{~nm}$ were used for DAPI and LC3 or Vps26 observations. To detect plasmon scattering from the MPNBs, the sample was irradiated using a laser wavelength of $559 \mathrm{~nm}$.

Magnetic Separation Protocol for Autophagosomes and Sample Preparation. The COS-1 cells (550 000) were seeded into $10 \mathrm{~cm}$-culture dishes in DMEM containing $10 \%$ FBS and incubated at $37^{\circ} \mathrm{C}$ overnight. The next day, $10 \mu \mathrm{L}$ of Lipofectamine 2000 was mixed with $500 \mu \mathrm{L}$ of Opti-MEM. After $5 \mathrm{~min}, 500 \mu \mathrm{L}$ of Opti-MEM containing $40 \mu \mathrm{g}$ of MPNBs was added, and the mixture was incubated for $20 \mathrm{~min}$. Then, 1 $\mathrm{mL}$ of the mixture was added into each dish. After incubation for $0 \mathrm{~min}(10 \mathrm{~s}), 15 \mathrm{~min}, 30 \mathrm{~min}, 1 \mathrm{~h}, 2 \mathrm{~h}, 4 \mathrm{~h}, 6 \mathrm{~h}$, and $8 \mathrm{~h}$ at $37{ }^{\circ} \mathrm{C}$, the culture medium was aspirated. The cells were washed with $10 \mathrm{~mL}$ of ice cold PBS twice; then, $1 \mathrm{~mL}$ of PBS containing protease inhibitor cocktail was added. The COS-1 cells were harvested from the dish using a cell lifter on ice. Then, the cell dispersion was transferred into a Dounce tissue grinder, and a homogenate was obtained in 20 strokes using a loose glass pole on ice. Then, the homogenate was subjected to magnetic separation using an autoMACS Pro Separator. The separation program was selected as "Posseld" in which $0.5 \mathrm{~mL}$ of the PS (magnetically attracted fraction) is obtained. Then, the PS was centrifuged at $1000 \mathrm{~g}$ for $5 \mathrm{~min}$ at $4{ }^{\circ} \mathrm{C}$. The running buffer was gently removed, and $200 \mu \mathrm{L}$ of PBS was added to disperse the precipitate to obtain the autophagosome-enriched MS fractions. Then, $200 \mu \mathrm{L}$ of freshly prepared sample buffer (50 $\mu \mathrm{L}$ of 2 -mercaptoethanol and $950 \mu \mathrm{L}$ of $2 \times$ Laemmli sample buffer) was added to the autophagosome-enriched fraction, and the sample was heated at $95{ }^{\circ} \mathrm{C}$ for $10 \mathrm{~min}$. The samples were separated into several tubes and stored at $-20{ }^{\circ} \mathrm{C}$ until use. For the control experiment, the sample was prepared by the same protocol as the homogenate with a $2 \mathrm{~h}$ incubation in the presence of transfection reagent only.

Sample Preparation for TEM Observation of MPNBIncorporated Autophagosomes. The cells were incubated for $30 \mathrm{~min}$ or $2 \mathrm{~h}$ with transfected MPNBs. After washing in PBS twice, the cells were fixed in phosphate-buffered $2 \%$ glutaraldehyde at $4{ }^{\circ} \mathrm{C}$ and subsequently postfixed in $2 \%$ osmium tetra-oxide for $3 \mathrm{~h}$ in an ice bath. Then, the specimens were dehydrated in graded ethanol and embedded in epoxy resin. Ultrathin sections were obtained by an ultramicrotome technique. Ultrathin sections stained with uranyl acetate for 10 min and lead-staining solution for 5 min were submitted for TEM observation.

SDS-PAGE and Western Blotting. The homogenate and MS fractions ( $50 \mu \mathrm{L}$ of each sample) were separated by SDSPAGE using a $15 \%$ polyacrylamide gel. A constant voltage was applied until the protein bands reached close to the end of the gel. Then, the gel was transferred to a polyvinylidene difluoride membrane (Bio-Rad) in CAPS buffer (10 mM CAPS and 10\% of methanol, $\mathrm{pH} 11$ ) by the semi-dry method. After blotting, the membrane was washed with Tris-buffered saline (TBS) containing 0.05\% TWEEN 20 (TBST) and blocked with 5\% skim milk in TBST for $30 \mathrm{~min}$ at room temperature. After washing the membrane with TBST, primary antibodies (against LC3, TfnR, LAMP2, and GAPDH) in 5\% BSA TBST were added and incubated at $4{ }^{\circ} \mathrm{C}$ overnight. The next day, the membrane was washed with TBST, and then secondary antibody conjugated to horseradish peroxidase was added and incubated for $1 \mathrm{~h}$ at room temperature. After washing with TBST, immunoreactive bands were detected using a chemiluminescence detector (LAS-3000, Fujifilm, Tokyo, Japan).

\section{ASSOCIATED CONTENT}

\section{Supporting Information}

The Supporting Information is available free of charge on the ACS Publications website at DOI: 10.1021/acsomega.7b00929.

Histogram of hydrodynamic diameter for the $\mathrm{Ag} / \mathrm{FeCo} /$ Ag core/shell/shell MPNBs dispersed in water and in Opti-MEM and cell viability assessment of COS-1 cells treated with increasing concentrations of MPNBs using intracellular esterase activity (PDF)

\section{AUTHOR INFORMATION}

\section{Corresponding Author}

*E-mail: shinya@jaist.ac.jp. Phone: +81-761-51-1611 (S.M.). ORCID

Kazuaki Matsumura: 0000-0001-9484-3073

Shinya Maenosono: 0000-0003-2669-8219

Notes

The authors declare no competing financial interest.

\section{ACKNOWLEDGMENTS}

This work was supported by the JSPS KAKENHI under grant nos. 15J10127 and 26600053. We are indebted to Prof. Y. Hiratsuka, K. Kawamoto, and C. Tatsumi of Japan Advanced Institute of Science and Technology (JAIST) for their assistance in cell experiments. We are grateful to Dr. S. Nakamura and Prof. K. Fujimoto of JAIST for their support in performing chemiluminescence detection of western blots. We also thank Prof. S. Waguri (Department of Anatomy and Histology, Fukushima Medical University) for his valuable comments regarding this manuscript.

\section{ABBREVIATIONS}

TEM, transmission electron microscopy; MPNB, magneticplasmonic hybrid nanobead; HR-TEM, high-resolution TEM; STEM-HAADF, scanning TEM equipped with a high-angle annular dark-field detector; EDS, energy-dispersive X-ray spectroscopy; LSPR, localized surface plasmon resonance; CLSM, confocal laser scanning microscopy; PLL, $\varepsilon$-poly-Llysine; PLL-SH, thiol group introduced PLL; ${ }^{1} \mathrm{H}$ NMR, proton nuclear magnetic resonance; BSA, bovine serum albumin; PBS, phosphate-buffered saline; DMEM, Dulbecco's modified Eagle's medium; FBS, fetal bovine serum; PS, positive selection; NS, negative selection; MS, magnetically separated; LC3, microtubule-associated protein light chain 3; Vps26, vacuolar protein sorting-associated protein 26; TfnR, transferrin receptor; LAMP2, lysosome-associated membrane protein 2; GAPDH, glyceraldehyde 3-phosphate dehydrogenase

\section{REFERENCES}

(1) Wu, L.-L.; Wen, C.-Y.; Hu, J.; Tang, M.; Qi, C.-B.; Li, N.; Liu, C.; Chen, L.; Pang, D.-W.; Zhang, Z.-L. Nanosphere-based one-step 
strategy for efficient and nondestructive detection of circulating tumor cells. Biosens. Bioelectron. 2017, 94, 219-226.

(2) Li, J.; Chen, L.; Zhang, X.; Zhang, Y.; Liu, H.; Sun, B.; Zhao, L.; Ge, N.; Qian, H.; Yang, Y.; Wu, M.; Yin, Z. Detection of circulating tumor cells in hepatocellular carcinoma using antibodies against asialoglycoprotein receptor, carbamoyl phosphate synthetase 1 and pan-cytokeratin. PLoS One 2014, 9, No. e96185.

(3) Taguchi, T.; Pypaert, M.; Warren, G. Biochemical subfractionation of the mammalian Golgi apparatus. Traffic 2003, 4, 344-352.

(4) Richardson, P. J.; Luzio, J. P. Immunoaffinity purification of membrane fractions from mammalian cells. Subcell. Biochem. 1988, 12, 221-241.

(5) Howell, K.-E.; Gruenberg, J.; Ito, A.; Palade, G.-E. Immunoisolation of subcellular components. Prog. Clin. Biol. Res. 1988, 270, $77-90$.

(6) Luzio, J. P.; Newby, A. C.; Hales, C. N. A rapid immunological procedure for the isolation of hormonally sensitive rat fat-cell plasma membrane. Biochem. J. 1976, 154, 11-21.

(7) Ito, A.; Palade, G. E. Presence of NADPH-cytochrome P-450 reductase in rat liver Golgi membranes: Evidence obtained by immunoadsorption method. J. Cell Biol. 1978, 79, 590-597.

(8) de Curtis, I.; Howell, K. E.; Simons, K. Isolation of a fraction enriched in the trans-Golgi network from baby hamster kidney cells. Exp. Cell Res. 1988, 175, 248-265.

(9) Salamero, J.; Sztul, E. S.; Howell, K. E. Exocytic transport vesicles generated in vitro from the trans-Golgi network carry secretory and plasma membrane proteins. Proc. Natl. Acad. Sci. U.S.A. 1990, 87, $7717-7721$.

(10) Li, H.-S.; Stolz, D. B.; Romero, G. Characterization of endocytic vesicles using magnetic microbeads coated with signalling ligands. Traffic 2005, 6, 324-334.

(11) Nakamura, N.; Lill, J. R.; Phung, Q.; Jiang, Z.; Bakalarski, C.; de Mazière, A.; Klumperman, J.; Schlatter, M.; Delamarre, L.; Mellman, I. Endosomes are specialized platforms for bacterial sensing and NOD2 signalling. Nature 2014, 509, 240-244.

(12) Pearse, B. M. Clathrin: a unique protein associated with intracellular transfer of membrane by coated vesicles. Proc. Natl. Acad. Sci. U.S.A. 1976, 73, 1255-1259.

(13) Conner, S. D.; Schmid, S. L. Regulated portals of entry into the cell. Nature 2003, 422, 37-44.

(14) Chithrani, B. D.; Chan, W. C. W. Elucidating the mechanism of cellular uptake and removal of protein-coated gold nanoparticles of different sizes and shapes. Nano Lett. 2007, 7, 1542-1550.

(15) Williams, S. K. R.; Lee, H.; Turner, M. M. Size characterization of magnetic cell sorting microbeads using flow field-flow fractionation and photon correlation spectroscopy. J. Magn. Magn. Mater. 1999, 194, 248-253.

(16) Takahashi, M.; Mohan, P.; Nakade, A.; Higashimine, K.; Mott, D.; Hamada, T.; Matsumura, K.; Taguchi, T.; Maenosono, S. Ag/ $\mathrm{FeCo} / \mathrm{Ag}$ core/shell/shell magnetic nanoparticles with plasmonic imaging capability. Langmuir 2015, 31, 2228-2236.

(17) Maenosono, S.; Saita, S. Theoretical assessment of FePt Nanoparticles as heating elements for magnetic hyperthermia. IEEE Trans. Magn. 2006, 42, 1638-1642.

(18) Wang, J.; Yu, X.; Boriskina, S. V.; Reinhard, B. M. Quantification of differential ErbB1 and ErbB2 cell surface expression and spatial nanoclustering through plasmon coupling. Nano Lett. 2012, 12, 32313237.

(19) Wang, H.; Shen, J.; Li, Y.; Wei, Z.; Cao, G.; Gai, Z.; Hong, K.; Banerjee, P.; Zhou, S. Porous carbon protected magnetite and silver hybrid nanoparticles: morphological control, recyclable catalysts, and multicolor cell imaging. ACS Appl. Mater. Interfaces 2013, 5, 94469453.

(20) Fujita, N.; Morita, E.; Itoh, T.; Tanaka, A.; Nakaoka, M.; Osada, Y.; Umemoto, T.; Saitoh, T.; Nakatogawa, H.; Kobayashi, S.; Haraguchi, T.; Guan, J.-L.; Iwai, K.; Tokunaga, F.; Saito, K.; Ishibashi, K.; Akira, S.; Fukuda, M.; Noda, T.; Yoshimori, T.
Recruitment of the autophagic machinery to endosomes during infection is mediated by ubiquitin. J. Cell Biol. 2013, 203, 115-128.

(21) Kobayashi, S.; Kojidani, T.; Osakada, H.; Yamamoto, A.; Yoshimori, T.; Hiraoka, Y.; Haraguchi, T. Artificial induction of autophagy around polystyrene beads in nonphagocytic cells. Autophagy 2010, 6, 36-45.

(22) Takahashi, M.; Higashimine, K.; Mohan, P.; Mott, D. M.; Maenosono, S. Formation mechanism of magnetic-plasmonic Ag@ FeCo@Ag core-shell-shell nanoparticles: fact is more interesting than fiction. CrystEngComm 2015, 17, 6923-6929.

(23) Takahashi, M.; Mohan, P.; Mott, D. M.; Maenosono, S. Exchange bias in $\mathrm{Ag} / \mathrm{FeCo} / \mathrm{Ag}$ core/shell/shell nanoparticles due to partial oxidation of FeCo intermediate shell. J. Magn. Magn. Mater. 2016, 401, 339-344.

(24) Takahashi, M.; Mohan, P.; Higashimine, K.; Mott, D. M.; Maenosono, S. Transition of exchange bias from the linear to oscillatory regime with the progression of surface oxidation of Ag@ FeCo@Ag core@shell@shell nanoparticles. J. Appl. Phys. 2016, 120, 134301.

(25) Shih, I.-L.; Shen, M.-H.; Van, Y.-T. Microbial synthesis of poly $(\varepsilon$-lysine $)$ and its various applications. Bioresour. Technol. 2006, 97, $1148-1159$.

(26) Matsumura, K.; Hayashi, F.; Nagashima, T.; Hyon, S. H. Longterm cryopreservation of human mesenchymal stem cells using carboxylated poly-l-lysine without the addition of proteins or dimethyl sulfoxide. J. Biomater. Sci., Polym. Ed. 2013, 24, 1484-1497.

(27) Shyue, J.-J.; De Guire, M. R.; Nakanishi, T.; Masuda, Y.; Koumoto, K.; Sukenik, C. N. Acid-base properties and zeta potentials of self-assembled monolayers obtained via in situ transformations. Langmuir 2004, 20, 8693-8698.

(28) Xu, H.; Suslick, K. S. Water-soluble fluorescent silver nanoclusters. Adv. Mater. 2010, 22, 1078-1082.

(29) Yuan, X.; Luo, Z.; Zhang, Q.; Zhang, X.; Zheng, Y.; Lee, J. Y.; $\mathrm{Xie}, \mathrm{J}$. Synthesis of highly fluorescent metal $(\mathrm{Ag}, \mathrm{Au}, \mathrm{Pt}$, and $\mathrm{Cu})$ nanoclusters by electrostatically induced reversible phase transfer. ACS Nano 2011, 5, 8800-8808.

(30) Huang, S.; Pfeiffer, C.; Hollmann, J.; Friede, S.; Chen, J. J.-C.; Beyer, A.; Haas, B.; Volz, K.; Heimbrodt, W.; Martos, J. M. M.; Chang, W.; Parak, W. J. Synthesis and characterization of colloidal fluorescent silver nanoclusters. Langmuir 2012, 28, 8915-8919.

(31) Aslan, K.; Lakowicz, J. R.; Geddes, C. D. Plasmon light scattering in biology and medicine: new sensing approaches, visions and perspectives. Curr. Opin. Chem. Biol. 2005, 9, 538-544.

(32) Strømhaug, P. E.; Berg, T. O.; Fengsrud, M.; Seglen, P. O. Purification and characterization of autophagosomes from rat hepatocytes. Biochem. J. 1998, 335, 217-224.

(33) Seglen, P. O.; Brinchmann, M. F. Purification of autophagosomes from rat hepatocytes. Autophagy 2010, 6, 542-547.

(34) Uematsu, M.; Nishimura, T.; Sakamaki, Y.; Yamamoto, H.; Mizushima, N. Accumulation of undegraded autophagosomes by expression of dominant-negative STX17 (syntaxin 17) mutants. Autophagy 2017, 1-13.

(35) Kneipp, J.; Kneipp, H.; Wittig, B.; Kneipp, K. One- and twophoton excited optical $\mathrm{pH}$ probing for cells using surface-enhanced Raman and hyper-Raman nanosensors. Nano Lett. 2007, 7, 28192823.

(36) Maxfield, F. R.; McGraw, T. E. Endocytic recycling. Nat. Rev. Mol. Cell Biol. 2004, 5, 121-132.

(37) Sandvig, K.; van Deurs, B. Membrane traffic exploited by protein toxins. Annu. Rev. Cell Dev. Biol. 2002, 18, 1-24. 